intense light from time to time arise in those portions of the tail that were furthest from the nucleus. Sometimes instantaneous, and appearing upon a small extension of the extremity of the tail, which then became more visible, the fugitive gleams reminded me of the pulsations of the aurora borealis. At other times they were less fleeting, and their propagation in rapid succession could be followed for some seconds in the direction of the nucleus near the extremity of the tail. These appearances then resembled the progressive undulations of the aurora borealis, but even in this case they were only visible in the last third of the length of the tail. The gleams in question were similar to those that I remember to have seen in the tail of the great comet of 1843 , and which were observed by very many astronomers." "

The American observers of Donati's comet in 1868 described a number of brignter bands "like auroral streamers" crossing the tail and diverging from a point between the nucleus and the sun. ${ }^{1}$

This point is one well worthy of subsequent inquiry. I have brought together evidence to show that in the aurora one of the chiet factors in the production of the spectrum is meteoric dust.

If this be conceded, we have meteoric dust in all probability very slowly falling through our atmosphere at a height at which its pressure is very low, the luminosity both of the dust and the atmosphere being produced by electricity. Whether the electricity is produced by the movement is a matter on which at present we are quite ignorant, but if it be eventually shown that all auroræ follow well-recognized star-showers a certain amount of plausibility will be accorded to the notion.

However this may be, we must in the case of the aurora regard the permanent gases in the air as a constant, and the dust as the variable.

But if we wish to assimilate these displays with comets' tails, we must in the latter case consider meteorites in space as the constant, and the permanent gases rèpelled from the comet as the variable.

Prof. Tait, assuming that the head of a comet is a swarm of meteorites or stones, varying in size from a marble to boulders 20 or 30 feet in diameter, has shown that all the various cometary phenomena may be explained. His researches have not yet been printed in extenso, but the following general statement gives a summary of the results of his calculations which appeared in Good Words some time ago.

Firstly, with regard to the masses of the comets. The total mass of a comet cannot be very great, for, as we have seen, no measurable disturbance of planetary orbits has been known to be produced, and this small mass is just as likely to be due to scattered solid masses as to one continuous gaseous mass, and indeed we know that this is so. In the case of comets of but small masses, the component meteorites would be small and far apart. Then with regard to the transparency of the comet, it is calculated that a meteorite 25 feet in diameter at a distance of half a million miles from us conld not totally eclipse a star of the same size as our sun, even if it were at such a distance as to be barely visible to the naked eye. Again, if some of the meteorites were large enough to eclipse the stars behind the comet, the eclipse would be of very brief duration, and we should see the star as if nothing had happened. In order for the comet to re duce the light of a star seen through it by one-tenth, it would require to be 300 miles thick, supposing the stones to be I inch cube and 20 feet apart.

While the swarm which builds up the comet is coursing round the sun as a whole, the individual members will themselves gravitate towards each other; and if we suppose the whole mass to be I/IOOO that of the earth, and the meteorites to be uniformly distributed in a sphere 20,000 miles in diameter, those coming from the outside to the centre of the group would have a velocity of about 500 fect per second. The stones colliding will generate heat, and some gas will be evolved; some members of the mass will be quickened, while other constituents of the mass will be retarded in their motion, and in this way we have a probably sufficient explaration of the various forms which the telescope has revealed to us. And then finally Prof. Tait goes on to show that the result of these collisions would be such a smashing up of the constituents of the swarm that much finely-attenuated material would be left behind, sufficient to reflect sunlight, and to give rise to the phenomena of the tail.

Webb, p. 197 .
If in the imaginary swarm the mass of each stone be 100 pounds, and its velocity, due to attraction, be 500 feet per second, the heat resulting from the impact of two of them would be quite sufficient to volatilize a portion, and to make the outsides of the stones white-hot. Stones of this weight would be about ro inches cube, and in the swarm considered there would therefore be about I36,000,000,000,000,000,000 of them. At the rate of one collision per second, there being about $31,436,000$ seconds in a year, there would be a possibility of one collision per second for $2,150,000,000,000$ years. There would therefore be material for such collisions for a period of over two million years even at the extravagant rate of one million per second, and on the assumption that no stone comes into collision with another more than once.

The whole mass being $x / 1000$ that of the earth, and the space occupied being 250 times that occupied by the earth, the stones in question being $\mathrm{I} O$ inches cube will only occupy about $\mathrm{I} / 8000$ of the space through which they are distributed; the average distance apart would be about I 7 feet. The swarm would reflect about half as much sunlight as a slab of the same material in the same place, but it would probably be too opaque to transmit starlight. By making the stones larger, and thus increasing the distances between them, the luminosity would be retained, while at the same time the swarm would be sufficiently transparent. It thus seems to suit the hypothesis better if we regard the separate stones to be greater than Io inches cube.

J. NORMAN LOCKYER.

(To be continued.)

\section{THE FORCES OF ELECTRIC OSCILLATIONS TREATED ACCORDING TO MAXWELL'S THEORY. BY DR. H. HERTZ.'}

I.

\section{Note by the Translator.}

THE early part of the following paper is no doubt familiar to the more important persons in this country, and therefore need perhaps hardly have been translated. N evertheless, as these experiments of Hertz form a sort of apotheosis of Maxwell's theory, it is natural to reprodice this portion as well as the rest ; and inasmuch as Hertz did not at first express his discoveries in Maxwellian language, it is interesting to see how he regards the matter since his conversion, and how he now presents his ideas to foreigners.

I have translated the paper because it seems to me a remarkable example of clear theoretic insight in conjunction with great experimental skill, because it is pleasantly written, and because it deals in a powerful manner with a profoundly interesting subject.

I can hardly hope to have escaped errors in translating, but the original paper in Wiedemann's Annalen for January of this year is very accessible. Oliver J. LODGe.

The results of the experiments on quick electric oscillation which I have carried out appear to me to lend to Maxwell's theory of electrodynamics an ascendancy over all others. At first I interpreted these experiments in terms of older notions, seeking to explain the phenomena in part by means of the cooperation of electrostatic and electro-magnetic forces. To Maxwell's theory in its pure development such a distinction is foreign. I wish, therefore, now to show that the phenomena can also be explained in terms of Maxwell's theory without any such distinction. If this attempt succeeds, questions about special propagation of electrostatic force, being meaningless in Maxwell's theory, are at once settled. And besides this special aim, a closer insight into the play of forces concerned in rectilinear oscillations is not without interest.

\section{The Formule.}

In what follows we have only to concern ourselves with forces in free ether. Lef $X, Y, Z$, be the components of electric force acting on the points $x, y, z$; let $\mathrm{I}, \mathrm{M}, \mathrm{N}$ be the corresponding components of magnetic force; let $t$ be the time, and let $\mathrm{A}$ stand for $\sqrt{ }(\mu \mathrm{K})$. Then, according to Maxwell, the time-rate of change of the forces is dependent on their distribution in space in the following way :-

I Translated and communicated by Dr. Oliver Lodge. 


$$
\begin{aligned}
& \mathrm{A} \frac{d \mathrm{~L}}{d t}=\frac{d \mathrm{Z}}{d y}-\frac{d \mathrm{Y}}{d z} \\
& \mathrm{~A} \frac{d \mathrm{M}}{d t}=\frac{d \mathrm{X}}{d z}-\frac{d \mathrm{Z}}{d x} \\
& \text {... (I) } \\
& \mathrm{A} \frac{d \mathrm{~N}}{d t}=\frac{d \mathrm{Y}}{d x}-\frac{d \mathrm{X}}{d y} \\
& \mathrm{~A} \frac{d \mathrm{X}}{d t}=\frac{d \mathrm{M}}{d z}-\frac{d \mathrm{~N}}{d y} \\
& \mathrm{~A} \frac{d \mathrm{X}}{d t}=\frac{d \mathrm{~N}}{d x}-\frac{d \mathrm{~L}}{d z} \\
& \mathrm{~A} \frac{d \mathrm{Z}}{d t}=\frac{d \mathrm{~L}}{d y}-\frac{d \mathrm{M}}{d x}
\end{aligned}
$$

Originally, and therefore always, the following conditions must be satisfied:

$$
\frac{d \mathrm{~L}}{d x}+\frac{d \mathrm{M}}{d y}+\frac{d \mathrm{~N}}{d z}=0 \text {, and } \frac{d \mathrm{X}}{d x}+\frac{d \mathrm{Y}}{d y}+\frac{d \mathrm{Z}}{d z}=0 .
$$

The electric energy contained in a portion of ether of volume $\tau$ is-

the magnetic energy is-

$$
\frac{\mathbf{I}}{8 \pi} \int\left(\mathrm{X}^{2}+\mathrm{Y}^{2}+\mathrm{Z}^{2}\right) d \tau
$$

$$
\frac{\mathbf{I}}{8 \pi} \int\left(\mathrm{L}^{2}+\mathrm{M}^{2}+\mathrm{N}^{2}\right) d \tau
$$

the integration extending all through the volume. The total energy is the sum of both these portions.

These expressions form the essential ingredients of Maxwell's theory as it relates to the ether. Maxwell arrived at them by forsaking action at a distance, and by accummodating the ether with the properties of a highly dielectric medium. One can also get the same equations in another way. But hitherto no direct proof of the validity of these equations has been afforded by experience. It áppears most logical, therefore, to regard them independently of any way in which they may have been arrived at, to consider them as hypothetical assumptions, and to let their probability depend upon the very great number of legitimate conclisions which they embrace. Taking this point of view, one can do without a series of auxiliary ideas, which render the understanding of Maxwell's theory more difficult, even if on no other ground than that, so soon as one finally excludes the bypothesis of immediate action at a distance, these notions possess no meaning.

Multiply equations (I) by $\mathrm{L}, \mathrm{M}, \mathrm{N}$, and (2) by X, Y, Z ; add the equations together, and integrate over the whole space, whose volume element is $d \tau$, and whose surface element is $d \mathrm{~S}$; we get-

$$
\begin{gathered}
\frac{d}{d t}\left\{\mathrm{E}_{e}+\mathrm{E}_{m}\right\} \\
=\frac{\mathrm{I}}{4 \pi \mathrm{A}} \int\left\{(\mathrm{NY}-\mathrm{MZ}) \lambda+(\mathrm{L} Z-\mathrm{NX}) \mu+(\mathrm{MX}-\mathrm{LY}) \nu_{j}^{2} d \mathrm{~S},\right.
\end{gathered}
$$

where $\lambda, \mu, \nu$ are the direction-cosines of the normal to the surface.

This equation shows that the amount by which the energy of the space has increased can be regarded as having entered through its walls. The quantity entering through any single element of surface is equal to the product of the components of the electric and magnetic forces which belong to that element, multiplied by the sine of the angle between them, and divided by $4 \pi \mathrm{A}$. On this result it is well known that Prof. Poynting has founded a remarkable theory on the transfer of energy in the electro-magnetic field.

For the purpose of solving the equation, we limit ourselves to the special but important case where the distribution of the electric force is symmetrical about the axis of $z$, and hence that this force is absent at every point of the meridian planes intersecting in the axis of $z$, and only depends on the $z$ co-ordinate of a point, and on its distance, $r=\sqrt{ } x^{2}+y^{2}$, from the $z$ axis. We will denote the electric force in the direction of $r$, namely, $\mathrm{X}_{\frac{x}{r}}^{x}+\mathrm{Y}_{r}^{y}$, by $\mathrm{R}$; and the component of the magnetic force which is normal to the meridian planes, viz. $\mathrm{L} \frac{y}{r}-\mathrm{M}_{\frac{y}{r}}^{y}$, by $\mathrm{P}$. We assert further that if $\Pi$ is any function of $r, z$, and $t$, which satisfies the equation-

$$
\mathrm{A}^{2} \frac{d^{2} \Pi}{d t^{2}}=\nabla \Pi
$$

and if we put $\mathrm{Q}=r \cdot \frac{d \Pi}{d r}$, the following is a possible solution of our equations :-

$$
\begin{aligned}
& \mathrm{Z}=\frac{\mathrm{I}}{r} \frac{\mathrm{Q}}{d r}, \\
& \mathrm{R}=-\frac{\mathrm{r}}{r} \frac{d \mathrm{Q}}{i t}, \\
& \mathrm{P}=\frac{\mathrm{A}}{r} \frac{d \mathrm{Q}}{\mathrm{r}} \mathrm{dt}, \\
& \mathrm{N}=\mathrm{o} .
\end{aligned}
$$

To prove this assertion we observe that

$$
\begin{aligned}
& \mathrm{X}=\mathrm{R} \frac{d r}{d x}=-\frac{d^{2} \Pi}{d x d z}, \\
& \mathrm{Y}=\mathrm{R} \frac{d r}{d y}=-\frac{d^{2} \Pi}{d y d z}, \\
& \mathrm{Z}=\mathrm{I} \frac{d}{d r}\left(r \frac{d \Pi}{d r}\right)=\frac{d^{2} \Pi}{d x^{2}}+\frac{d^{2} \Pi}{d y^{2}}, \\
& \mathrm{~L}=\mathrm{P} \begin{array}{l}
d r \\
d y
\end{array}=\mathrm{A} \frac{d^{2} \Pi}{d y d t}, \\
& \mathrm{M}=-\mathrm{P} d r=-\mathrm{A} \frac{d^{2} \Pi}{d x d t}, \\
& \mathrm{~N}=\mathrm{o} .
\end{aligned}
$$

One has only to insert these expressions into equations (1), (2), (3), to find equations (2) and (3) identically satisfied, and (I) also if we have regard to the differential equation for $\Pi$.

It may be mentioned that also inversely, neglecting certain practically unimportant limitations, every possible distribution of electric force which is symmetrical to the axis of $z$ can be represented in the above form, but it is not necessary for the sequel to substantiate this assertion.

The function $Q$ is of importance. The lines in which the surface of rotation $Q=$ const. cut the meridian planes are the lines of electric force; the construction of the same for one meridian plane furnishes at every instant an immediate presentation of the force distribution.

If we cut the shell between $\mathrm{Q}$ and $\mathrm{Q}+d \mathrm{Q}$ by a surface of rotation round the axis of $z$, the product of electric force and surface which Maxwell calls the "induction" is for every such surface the same. If we arrange the system of surfaces $Q=$ const. in such a way that $Q$ increases in arithmetic progression, the same statement remains true when we compare the sections of the different shells with one another.

In the plane diagram which consists of sections of the meridian plane with the equidistant surfaces $Q=$ constant, the electric force is inversely proportional to the normal distance of consecutive lines $\mathrm{Q}=$ const. only for the case when points compared lie at the same distance from the axis of $z$. In general the rule is that the force is inversely proportional to the product of this distance and of the co-ordinate $r$ of the point considered.

If we introduce polar co-ordinates $\rho$ and $\theta$ they will be like this.

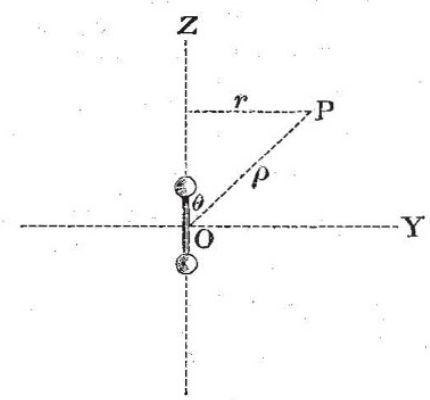

The figure represents an electric oscillator at origin of c3-ordinates as intended to be understood by Hertz.

The Forces concerned in a Rectilinear Oscillation.

Let $\mathrm{E}$ denote a quantity of electricity, and $l$ a lengtb; let $m=\frac{\pi}{\lambda}$ be a reciprocal length, and $n=\frac{\pi}{T}$ a reciprocal time; and let us put

$$
\Pi=\mathrm{E} \frac{l}{o} \sin (m \rho-n t) \text {. }
$$


This value satisfies the equation-

$$
\mathrm{A}^{2} \frac{d^{2} \Pi}{d t^{2}}=\nabla \Pi
$$

so soon as we settle that,

$$
\frac{m}{n}=\frac{\mathrm{T}}{\lambda}=\mathrm{A},
$$

and $\frac{\lambda}{\bar{T}}$ will be the velocity of light. And, indeed, the introduced equation is satisfied everywhere, except at the origin.

In order to find out what electrical processes are set up by the distribution of forces specified by $\Pi$, we investigate its immediate surroundings.

We put $\rho$ vanishing in comparison with $\lambda$, and neglect $m_{\rho}$ in comparison with $n t$.

Then-

$$
\Pi=\mathrm{E} \frac{l}{\rho} \sin n t .
$$

Since, now-

$$
\left(\frac{d^{2}}{d x^{2}}+\frac{d^{2}}{d y^{2}}\right)_{\rho}^{\mathbf{I}}=-\frac{d^{2}}{d z^{2}}\left(\frac{\mathrm{I}}{\rho}\right)
$$

we have-

$$
\mathrm{X}=-\frac{d^{2} \Pi}{d x d z}, \quad \mathrm{Y}=-\frac{d^{2} \Pi}{d y d z}, \quad \mathrm{Z}=-\frac{d^{2} \Pi}{d z d z} .
$$

So the electric forces appear as the derivative of a potential-

$$
\phi=\frac{d \Pi}{d z}=\mathrm{E} l \sin n t \cdot \frac{d}{d z}\left(\frac{\mathbf{I}}{\rho}\right) ;
$$

and this expresses an electrical [Doppelpunkt, by which I suppose is meant either an involution or a spherical harmonic] whose axis coincides with the $z$ axis, and whose moment oscillates between the extreme values $\mathrm{E} l$ and $-\mathrm{E} l$ with the period $\mathrm{T}$.

Our force distribution, therefore, represents the action of a rectilinear oscillator which has the very small length $l$, and on whose poles at the maximum the quantities of electricity $\pm \mathrm{E}$ are free.

The magnetic force perpendicular to the direction of the oscillator is, in the immediate neighbourhood,

$$
\mathrm{P}=-\mathrm{AE} \ln \cos n t \frac{\sin \theta}{\rho^{2}} .
$$

According to the Biot-Savart law, this is the force of a current element in the direction of the axis of $z$, of length $l$, whose intensity, magnetically measured, oscillates between the extreme values $\pm \frac{\pi \mathrm{AE}}{\mathrm{T}}$. In fact the motion of the electricity $\mathrm{E}$ corresponds to a current of that magnitude.

From $I I$ we get-

$$
\mathrm{Q}=\mathrm{E} l m\left\{\cos (m \rho-n t)-\frac{\sin (m \rho-n t)}{m \rho}\right\} \sin ^{2} \theta,
$$

and from this the forces $Z, R, P$ follow by differentiation.

The formulæ are too complicated for it to be possible to obtain immediately from them in their general form a representation of the distribution of the forces. For some special cases the results are meanwhile proportionately simple. We get these at once-

(I) The immediate neighbourhood of the oscillator we hive already treated.

(2) In the $z$ axis, i.e. in the direction of swing, we have $d r=\rho d \theta, d z=d \rho, \theta=0$; so then-

$$
\begin{gathered}
\mathrm{R}=\mathrm{o}, \mathrm{P}=\mathrm{o}, \\
L=\frac{2 \mathrm{E} l m}{\rho^{*}}\left\{\cos (m \rho-n t)-\frac{\sin (m \rho-n t)}{m \rho}\right\} .
\end{gathered}
$$

The electric force acts always in the direction of the oscilla tor; it diminishes for small distances as the inverse cube, for greater distances as the inverse square, of the distance.

(3) In the $x y$ plane, or $z=0$, we have $d z=-\rho d \theta, d \rho=d r$, $\theta=90$, and so-

$$
\begin{gathered}
\mathrm{P}=\frac{\mathrm{AE} l m n}{r}\left\{\sin (m r-n t)+\frac{\cos (m r-n t)}{m r}\right\} \\
\mathrm{R}=\mathrm{o}, \\
\mathrm{Z}=\frac{\mathrm{E} l m^{2}}{r}\left\{-\sin (m r-n t)-\frac{\cos (m r-n t)}{m r}+\frac{\sin m r-n t)}{m^{2} r^{2}}\right\} .
\end{gathered}
$$

The electric force in the equatorial plane through the oscillator is parallel to the oscillation, its amplitude being -

$$
\frac{\mathrm{E} l}{r^{3}} \sqrt{\mathrm{r}-m^{2} r^{2}+m^{4} r^{4}} \text {. }
$$

The force decreases with distance, at first quickly as the inverse cube, later only slowly, and inversely as the distance itself. At great distances the action of the oscillator can only be noticed in the equatorial plane, not in the axis itself.

(4) At very great distances we can neglect higher powers of $r / \rho$, compared with lower ones. So we get at such distances-

whence-

$$
\mathrm{Q}=\mathrm{E} l m \cos (m \rho-n t) \sin ^{2} \theta,
$$

Thence follows-

$$
\begin{aligned}
& \mathrm{P}=\mathrm{A} \frac{\mathrm{E} l m n}{\rho} \sin (m \rho-n t) \sin \theta, \\
& \mathrm{Z}=-\frac{\mathrm{E} l m^{2}}{\rho} \sin (m \rho-n t) \sin ^{2} \theta, \\
& \mathrm{R}=\frac{\mathrm{E} l m^{2}}{\rho} \sin (m \rho-n t) \sin \theta \cos \theta .
\end{aligned}
$$

$$
\mathrm{Z} \cos \theta+\mathrm{R} \sin \theta=\mathrm{o} .
$$

The direction of the force is therefore at great distances every. where normal to the radius vector from the origin of force; the spreading out occurs in pure transverse waves. The magnitude of the force is equal to $\frac{\mathrm{E} l m^{2}}{\rho} \sin (m \rho-n t) \sin \theta$, and decreases at a constant distance from the origin towards either axis, being proportional to the distance from this latter.

$$
\text { ( To be continued.) }
$$

\section{UNIVERSITY AND EDUCATIONAL INTELLIGENCE.}

CAMBRIDGE.-A Report on the new Chemical Laboratory states that it has cost in all $£ 33,697$, an excess of $£ 3000$ above the estimate in 1885 . The fittings and machinery have cost $£ 1900$ more than was estimated, and the foundations had to be made stronger at a cost of $£ 450$. The Financial Board state that they have every reason to believe that the University has full value for the outlay. The building' and fittings are substantial and well adapted to their purpose. Prof. Liveing reports that the lecture-rooms are such that the audience can both see and hear, can sit comfortably and write with ease, and that the laboratori ss will bear comparison with the best of those to be found elsewhere.

$£ 200$ is asked for the purchase of additional apparatus for the Pathological Laboratory (the old Chemical Laboratory).

At Cavendish College eight scholarships of $£, 30$ per annum will be offered on July 23 next to students who will be under eighteen years old on October I next. One or more may be given for natural science (chemistry, physics, botany, geology, in all of which there will be a practical examination). Further particulars may be obtained from the Master of Cavendish College.

\section{SCIENTIFIC SERIALS.}

Bulletins de la Société d'Anthropologie de Paris, tome II, fasc. 3,1888 . - Continuations of M. G. Hervé's observations on the cerebral convolution known as "Broca's." Great importance attaches to the discovery by the late eminent M. Broca of this anatomical characteristic in man, which he found to be absent in all animals below the Anthropomorpha, and while it appears in the latter only in a simple and rudimentary form, it is fully developed in the human brain. This fact in itself gives support to the hypothesis that intermediate types, now lost, must have been interposed between man and the still existing forms of the Anthropomorpha, and yet more important are the results yielded by recent physical researches, which clearly show that the normal human brain possesses a quadruple system of the frontal convolutions due to the doubling of the binary frontal lobes, while in Broca's convolution we must, moreover, recognize the origin and function of speech and memory. In the microcephali, in idiots, deaf-mutes, and in all persons of inferior intelligence, this convolution is more or less atrophied, especially within the insula or centre, where it unites with the other frontal convolutions near the extremity of the olfactory channel. -Close of the statistical inquiry regarding the colours of the eyes and hair in France, by M. Topinard. After having collected the results of 180,000 observations, M. Topinard an- 